\title{
Making In Situ Whole-Cell Patch Clamp Recordings from Xenopus laevis Tadpole Neurons
}

\author{
Wen-Chang Li ${ }^{1}$ \\ 1 School of Psychology \& Neuroscience, Westburn Lane, St Andrews, Fife, KY16 9TS, United Kingdom, \\ tel: +441334463579, wl21@st-andrews.ac.uk
}

\begin{abstract}
Xenopus laevis tadpoles have been an excellent, simple vertebrate model for studying the basic organization and physiology of the spinal cord and motor centers in the brainstem. In the past, intracellular recordings from the spinal and brainstem neurons were primarily made using sharp electrodes, although whole-cell patch clamp technology has been around since the early 1980s. In this protocol, I describe the dissections and procedures needed for in situ whole-cell patch clamp recording, which has become routine in tadpole neurophysiology since the early 2000 s. The critical step in the dissections is to delicately remove some ependymal cells lining the tadpole neurocoele in order to expose clean neuronal somata without severing axon tracts. Whole-cell recordings can then be made from the somata in either current- or voltage-clamp mode.
\end{abstract}

\section{MATERIALS}

\section{Reagents}

3-aminobenzoic acid ester (MS-222, $0.1 \%$ in saline)

Store for a couple of weeks in a sealed container at room temperature.

a-bungarotoxin $\left(100 \mu \mathrm{M}\right.$ in distilled water; store at $-20^{\circ} \mathrm{C}$ in $100 \mu \mathrm{L}$ aliquots)

Dilute $100 \mu \mathrm{L}$ stock to $1 \mathrm{~mL}$ with saline. Store at $4^{\circ} \mathrm{C}$ for up to a couple of weeks between uses.

Intracellular pipette solution for Xenopus $<\mathrm{R}>$

$\mathrm{NaOH}(5 \mathrm{M})$

Saline $<\mathrm{R}>$

Xenopus laevis tadpoles, stage 37/38 (Nieuwkoop and Faber 1956)

\section{Equipment}

\section{Equipment for dissections}

Blunt tungsten needle

Prepare by dipping the end of a short piece of $200 \mu \mathrm{m}$ tungsten wire glued onto a handle into the $\mathrm{NaOH}$ etching solution (Li et al. 2014).

Cold-light source with light guides

Dissection bath with rotatable Sylgard stage (Fig.1A, (Li et al. 2002))

Dissection microscope with magnification up to 80-120 times

Electrical etching kit (24V, $1500 \mathrm{~mA} \mathrm{AC}$ adaptor, connected to a plastic dish filled with $5 \mathrm{M} \mathrm{NaOH}$ solution with a pair of forceps to hold needles and pins at one end of the electrical circuit)

This kit is used to make tungsten dissection needles and pins.

Fine forceps (Dumont Tweezers, WPI)

Electrically etched fine tungsten dissection needle

Forceps

These are used for moving pins.

Microcentrifuge tubes

Petri dish

Pins 
Prepare by dipping the end of a $50 \mu \mathrm{m}$ tungsten wire a few $\mathrm{mm}$ long into the $\mathrm{NaOH}$ etching solution (Li et al. 2014).

\section{Equipment for recordings}

For details on how the equipment is connected see Fig.1C.

Coarse manipulators (MX10; SD Instruments): for ventral root recording and stimulating electrodes.

Coverslip (50mm x 33mm, $0.17 \mathrm{~mm}$ thick)

Digitizer (CED power 1401 from Cambridge Electronic Design)

Electrical stimulator (DS3; Digitimer)

Extracellular amplifier (four-channel; A-M Systems)

This is used to amplify ventral root signals.

Motor-driven fine control manipulator (MX763OR manipulators; SD Instruments)

Nikon E600FN microscope with 4X and water immersion 40X or 60X objectives and a translation table

Patch-clamp amplifier (Multiclamp 700B; Molecular Devices)

Peristaltic pump

Pipette puller (P-97; Sutter Instrument)

Recording bath with rotatable Sylgard stage (Fig.1B, (Li et al. 2002))

Sampling software (Signal version 5 from Cambridge Electronic Design)

Single barrel capillary glass with microfilament for patch clamp (A-M systems)

Syringes

Three-way connectors

$0.2 \mu \mathrm{m}$ filters (Whatman Puradisc 13 syringe filters, Sigma)

Vaseline

Workstation PC

\section{METHOD}

\section{Dissections and tadpole preparation}

1. Anesthetize a stage $37 / 38$ tadpole (Nieuwkoop and Faber 1956) with $0.1 \%$ MS-222 for $\sim 20$ sec in a Petri dish at room temperature.

2. Pin the tadpole through its notochord at two locations onto the rotatable Sylgard stage in the dissection bath filled with saline illuminated by the cold light (Li et al. 2014).

3. Make a vertical cut in the tadpole dorsal fin near the hindbrain by crushing the skin against the Sylgard using the blunt tungsten needle. Extend the cut by sliding the needle tip inside the dorsal fin along the body axis.

4. Free the tadpole and transfer it to a microcentrifuge tube containing $1 \mathrm{~mL}$ saline with $10 \mu \mathrm{M} \mathrm{\alpha}$ bungarotoxin with a small transfer pipette. [Incubate for 20-30 $\mathrm{min}$ for immobilization at room temperature.

5. After immobilization, re-pin the tadpole onto the Sylgard stage in the dissection bath.

6. Remove the majority of the yolk belly in the trunk using a blunt dissection needle (Fig.1F).

7. Cut free the middle strip of skin connecting the head and tail skin using the fine dissection needle.

8. Peel off the skin strip using a pair of fine forceps to expose the swimming myotomes.

9. Push a blunt dissection needle between the spinal cord and the swimming muscles on the exposed side along the body axis. Cut one end of the loosened muscles. Remove the contracted lump of muscles using a pair of fine forceps. Remove the loose tissue using a pair of fine forceps or by rubbing the side of the dissection needle gently against the spinal surface.

10. Pierce the dorsal roof of the hindbrain and open up the half-exposed spinal cord by gently pushing the dissection needle inside the neurocoele (Fig. 1D). 
11. In order to expose more ventral spinal neurons, remove some ependymal cells lining the neurocoele (Fig.1D). Clear loose tissue by gently rubbing the side of a dissection needle against the exposed spinal somata.

12. Free the tadpole. Transfer it to the recording bath filled with saline (Fig.1B)and re-pin it onto the small rotatable Sylgard stage at its edge (Fig. 1F). Use Vaseline to attach a thin piece of coverslip to the bottom of the recording chamber to allow light transmission from below on the Nikon E600FN microscope. Leave a gap in the stage to avoid light distortion by Sylgard.

\section{Recordings}

See Fig. $1 C$ for recording set up.

13. Tilt the Sylgard stage and tadpole to an optimal angle for clear visualization of exposed somata in the spinal cord under the Nikon E600FN microscope (Fig. 1D-F).

14. Circulate saline in the recording chamber with a peristatic pump at $\sim 2 \mathrm{~mL}$ per min.

15. Fill the stimulating and ventral root suction electrodes with saline by pulling their connected syringes and place the electrodes on tadpole tail skin to start fictive swimming and muscle cleft to monitor fictive swimming using the coarse manipulators (Fig.1F).

16. Pull patch pipettes from the capillary glass using the p-97 puller to the desired shape (Fig.1E) and DC resistance 5-10 $\mathrm{M} \Omega$.

17. Pull a filler tube from a plastic pipette tip over a small flame. Attach the filler tube to a small syringe, with a $0.2 \mu \mathrm{m}$ filter, to back-fill a patch pipette with the intracellular solution.

18. Mount the patch pipette on the patch clamp amplifier head stage. Make sure the tubing connecting to the pipette is airtight. Apply gentle pressure $\left(7 \mathrm{~cm} \mathrm{H}_{2} \mathrm{O}\right)$ by compressing about 2 $\mathrm{mL}$ air trapped in the tubing by $10 \mu \mathrm{L}$ using another $1 \mathrm{~mL}$ syringe. Use a three-way connector to lock the pressure before the patch electrode touches the cell membrane.

19. Lower the pipette into the saline using the motorized manipulator and move it close to the exposed spinal cord with the $4 \mathrm{X}$ objective.

20. In the Multiclamp 700B controller software, choose the recording mode as current-clamp (IC) for the channel connected to the headstage holding the patch pipette.

21. Set up test step current injection pulses in Signal software outputs (2 ms with a gap of $3 \mathrm{~ms}$ and alternating polarities at $50 \mathrm{~Hz}, 100 \mathrm{pA}, \mathrm{Fig} .1 \mathrm{G}$ ) for monitoring the progression of the gigaohm seal and whole-cell recording.

22. Under the 40x water immersion lens, use the motorized manipulator to advance the patch pipette to contact an exposed neuron soma.

23. Neutralize electrode capacitance (stray capacitance) by turning up its reading in Multiclamp $700 \mathrm{~B}$ controller until the recording trace thickens but does not oscillate.

24. Once the pipette touches the soma, release the pressure and apply gentle suction to the pipette (14-35 $\mathrm{cm} \mathrm{H} \mathrm{H}_{2} \mathrm{O}$, expand $2 \mathrm{~mL}$ air by $20-50 \mu \mathrm{L}$ ). The voltage responses to test current injections should change from that resembling the step currents to monophasic with increased amplitude (Fig.1G). Very often the gigaohm seal forms within seconds.

25. Once a gigaohm seal is achieved, apply large zapping currents configured in Signal sfotware to rupture the membrane inside the pipette tip (2-5 nA, 2 to 5 pulses at $100 \mathrm{~Hz}$ and $2 \mathrm{~ms}$ in duration).

26. Determine if the membrane is ruptured as follows: if the membrane is ruptured and whole-cell recording achieved, test pulse responses will change instantly to smaller monophasic shapes (Fig.1G).

See Troubleshooting.

27. Balance bridge by turning up the series resistance value in the Multiclamp $700 \mathrm{~B}$ controller. 
28. Stimulate tadpole skin electrically to start fictive swimming (0.5-1 ms pulses, can be triggered using Signal output) and record intracellular activity in current-clamp mode (Fig.1H).

See Troubleshooting.

\section{TROUBLESHOOTING}

Problem (Step 26): Difficulty is encountered in rupturing the cellular membrane.

Solution: It can be difficult to rupture the cellular membrane if the osmolarity of the pipette solution is high relative to that of saline. Saline osmolarity should be $15-20 \mathrm{mOsm} / \mathrm{L} \mathrm{H}_{2} \mathrm{O}$ higher than that of pipette solution. If it is not, adjust the pipette solution osmolarity by adding sucrose or the saline osmolarity by changing $\mathrm{NaCl}$ concentration. Membrane rupture can also be difficult if the pipette has formed a false gigaohm seal with loose tissue or ependymal cells. Be sure to clean the exposed somata thoroughly in Step 11. Replace the pipette that has formed a false seal and avoid contacting loose tissue before touching soma membrane.

Problem (Step 28): A small action potential overshoot is observed.

Solution: Make sure capacitance neutralization is properly adjusted in Step 23 before whole-cell recordings. Series resistance can rise during recordings due to movement of yolk platelets (Fig.1E arrowheads). If this occurs, sudden suction can be applied to unclog the pipette by quickly pulling out the cork of the connected $1 \mathrm{~mL}$ syringe. If this fails, use a new patch pipette and re-patch the neuron.

\section{DISCUSSION}

Whole-cell recordings from tadpole neurons were first achieved in cultured spinal neurons (Ribera and Spitzer 1987; Dale 1991) but success with in situ recordings remained limited (Rohrbough and Spitzer 1996; Prime et al. 1999) until 2002 (Li et al. 2002). Because any membranous tissue can seal up the pipette, difficulty exists in generating a genuine gigaohm seal with a neuronal membrane. Fine dissections are required to expose somata to improve seal success. The need for surgical exposure of soma membrane has prevented its application to neurons deep in the CNS. It is also more difficult to see neurons in the brainstem where transmitted light is obscured by thicker tissue and Sylgard. Once established, a successful whole-cell recording can be sustained for several hours.

The conventional whole-cell recording can be adapted to expand its applications. For example, perforated patch-clamp recordings can be achieved by including amphotericin B or gramicidin (Kyrozis and Reichling 1995 ) in the pipette solution. Tadpole neuronal dendrites are short at stage $37 / 38$. This allows good space clamp to analyze voltage-dependent ionic currents and synaptic currents in voltage-clamp mode ( $\mathrm{Li}$ 2015). Neurobiotin or fluorescent dyes like Alexa Fluor 488 at $0.1 \%$ can also be added to the pipette solution to observe neuronal anatomy after fixation and processing or in situ (Li et al. 2002). New features in Signal 5 and 6 sampling software have recently been developed to enable dynamic clamp recordings to remove or add different "ion channels" and "synaptic connections" to the recorded neurons.

\section{REFERENCES}

Dale N. 1991. The Isolation and Identification of Spinal Neurons That Control Movement in the Xenopus Embryo. Eur J Neurosci 3: 1025-1035.

Kyrozis A, Reichling DB. 1995. Perforated-patch recording with gramicidin avoids artifactual changes in intracellular chloride concentration. J Neurosci Methods 57: 27-35.

Li WC. 2015. Selective Gating of Neuronal Activity by Intrinsic Properties in Distinct Motor Rhythms. J Neurosci 35: 9799-9810. 
Li WC, Soffe SR, Roberts A. 2002. Spinal inhibitory neurons that modulate cutaneous sensory pathways during locomotion in a simple vertebrate. J Neurosci 22: 10924-10934.

Li WC, Wagner M, Porter NJ. 2014. Behavioral observation of Xenopus tadpole swimming for neuroscience labs. J Undergrad Neurosci Educ 12: A107-113.

Nieuwkoop PD, Faber J. 1956. Normal tables of Xenopus laevis (Daudin), Amsterdam: North Holland.

Prime L, Pichon Y, Moore LE. 1999. N-Methyl-D-aspartate-induced oscillations in whole cell clamped neurons from the isolated spinal cord of Xenopus laevis embryos. J Neurophysiol 82: 1069-1073.

Ribera AB, Spitzer NC. 1987. Both barium and calcium activate neuronal potassium currents. Proc Natl Acad Sci U S A 84: 6577-6581.

Rohrbough J, Spitzer NC. 1996. Regulation of intracellular Cl- levels by $\mathrm{Na}(+)$-dependent $\mathrm{Cl}$ - cotransport distinguishes depolarizing from hyperpolarizing GABAA receptor-mediated responses in spinal neurons. J Neurosci 16: 82-91.

Winlove $\mathrm{Cl}$, Roberts A. 2012. The firing patterns of spinal neurons: in situ patch-clamp recordings reveal a key role for potassium currents. Eur J Neurosci 36: 2926-2940.

A

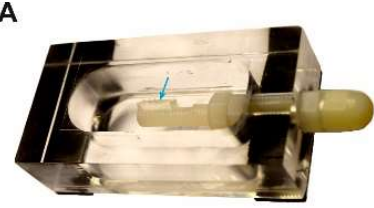

B

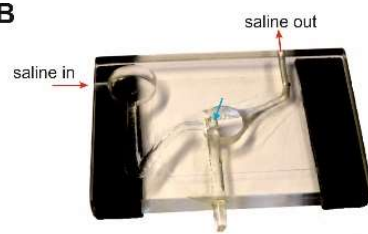

C
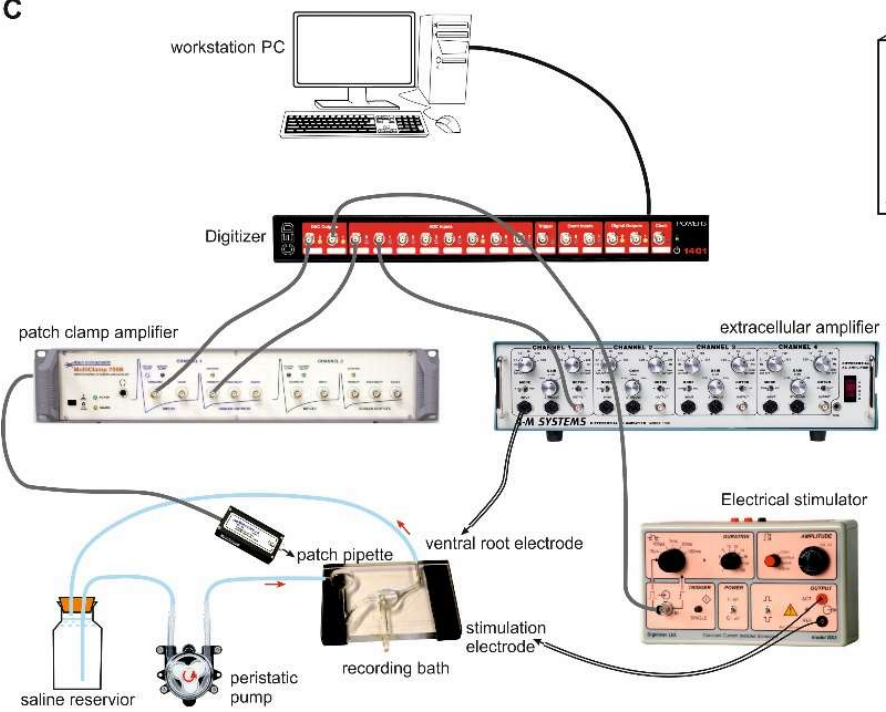

D

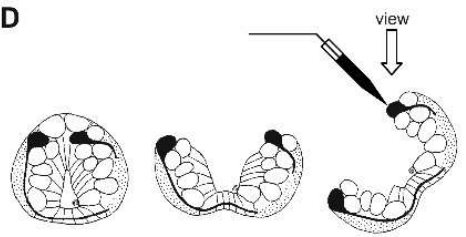

$\mathbf{F}$

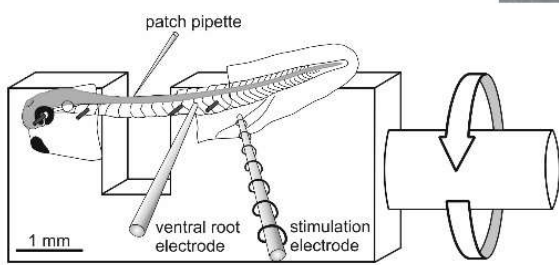

G
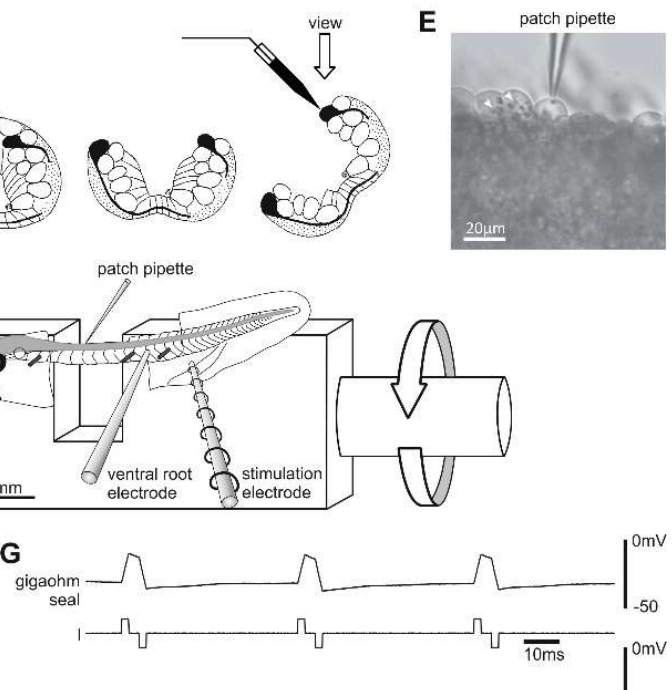
(1) 
C. The connections between the main equipment. Thick line to chassis means cable sockets are in the back panels. Electrodes, microscope and manipulators are omitted for simplicity. Red arrows in $\mathbf{B}$ and $\mathbf{C}$ indicate the direction of saline flow for the recording bath. D. A diagram of tadpole spinal cord cross sections showing neurons (ovals, two examples filled), ependymal cells (rectangles) and axon tracts (dotted area). The spinal cord is split open (middle diagram) and tilted to allow view of exposed neurons on the E600FN microscope after removal of ependymal cells (right diagram). E. A photo of a segment of the spinal cord after dissections in $\mathbf{D}$. The patch pipette is placed on a potential sensory Rohon-Beard neuron (dorsal is up, rostral is to the left). White arrow heads point at the yolk platelets inside neurons. $\mathbf{F}$. Arranging electrodes around a dissected tadpole (CNS shown grey) pinned at the edge of a rotatable Sylgard-lined platform over the gap, to evoke and record fictive swimming and make whole-cell recordings. G. Using brief current pulses (I) to monitor patch pipette seal with the cellular membrane and progression to whole-cell recordings. $\mathbf{H}$. The activity of a commissural interneuron (cIN) recorded in whole-cell current clamp mode at the beginning of a fictive swimming episode initiated by a brief electrical stimulus $(0.5 \mathrm{~ms}$, arrow). v.r. stands for ventral root. Solid short lines are pins through the notochord and myotomes. D and $\mathbf{F}$ are adapted from (Li et al. 2002). $\mathbf{E}$ is reprinted, with permission, from (Winlove and Roberts 2012), copyright John Wiley and Sons. 